\title{
Matrix Elements without Quark Masses on the Lattice
}

\author{
A. Donini ${ }^{\mathrm{a}}$, V. Giménez ${ }^{\mathrm{b}}$, L. Giusti $^{\mathrm{*} *}$, G. Martinelli. ${ }^{\mathrm{d}}$ \\ ${ }^{a}$ Dep. de Fisica Teorica, Univ. Autonoma de Madrid, Cantoblanco, E-28049 Madrid, Spain. \\ ${ }^{\text {b} D e p . ~ d e ~ F i s i c a ~ T e o r i c a ~ a n d ~ I F I C, ~ U n i v . ~ d e ~ V a l e n c i a, ~ E-46100 ~ B u r j a s s o t, ~ V a l e n c i a, ~ S p a i n . ~}$ \\ ${ }^{\mathrm{c}}$ Department of Physics, Boston University Boston, MA 02215 USA.
}

dDip. di Fisica, Univ. di Roma "La Sapienza" and INFN-Sezione di Roma, I-00185 Roma, Italy.

We introduce a new parameterization of four-fermion matrix elements which does not involve quark masses and thus allows a reduction of systematic uncertainties in physical amplitudes. As a result the apparent quadratic dependence of $\epsilon^{\prime} / \epsilon$ on $m_{s}(\mu)$ is removed. To simplify the matching between lattice and continuum renormalization schemes, we express our results in terms of Renormalization Group Invariant $B$-parameters which are renormalization-scheme and scale independent. As an application of our proposal, matrix elements of $\Delta I=3 / 2$ and SUSY $\Delta F=2(F=S, C, B)$ four-fermion operators have been computed.

\section{Introduction}

Since the original proposals of using lattice QCD to study hadronic weak decays [1] 3], substantial theoretical and numerical progress has been made: the main theoretical aspects of the renormalization of composite four-fermion operators are well understood [4]; the calculation of $K^{0}-\bar{K}^{0}$ mixing has reached a level of accuracy which is unpaired by any other approach 汭; increasing precision has been gained in the determination of the electro-weak penguin amplitudes necessary to the prediction of the $\mathrm{CP}$-violation parameter $\epsilon^{\prime} / \epsilon$ 810]; finally matrix elements of $\Delta S=2$ operators which are relevant to study FCNC effects in SUSY models have been computed [9,10]. Methods, symbols and results reported in this talk are fully described in [9,10].

\section{Matrix elements without quark masses}

The analysis of $K^{0}-\bar{K}^{0}$ mixing with the most general $\Delta S=2$ effective Hamiltonian requires the knowledge of matrix elements $\left\langle\bar{K}^{0}\left|O_{i}\right| K^{0}\right\rangle$ of parity conserving parts of the following operators

$$
\begin{aligned}
& O_{1}=\bar{s}^{\alpha} \gamma_{\mu}\left(1-\gamma_{5}\right) d^{\alpha} \bar{s}^{\beta} \gamma_{\mu}\left(1-\gamma_{5}\right) d^{\beta}, \\
& O_{2}=\bar{s}^{\alpha}\left(1-\gamma_{5}\right) d^{\alpha} \bar{s}^{\beta}\left(1-\gamma_{5}\right) d^{\beta}, \\
& O_{3}=\bar{s}^{\alpha}\left(1-\gamma_{5}\right) d^{\beta} \bar{s}^{\beta}\left(1-\gamma_{5}\right) d^{\alpha}, \\
& O_{4}=\bar{s}^{\alpha}\left(1-\gamma_{5}\right) d^{\alpha} \bar{s}^{\beta}\left(1+\gamma_{5}\right) d^{\beta},
\end{aligned}
$$

\footnotetext{
*Talk presented by L. Giusti. This research was supported in part under DOE grant DE-FG02-91ER40676.
}

$O_{5}=\bar{s}^{\alpha}\left(1-\gamma_{5}\right) d^{\beta} \bar{s}^{\beta}\left(1+\gamma_{5}\right) d^{\alpha}$.

On the lattice, matrix elements of weak fourfermion operators are computed from first principles. But, following the common lore, they are usually given in terms of the so-called $B$ parameters which measure the deviation of their values from those obtained in the Vacuum Saturation Approximation (VSA). For operators in (1), the $B$-parameters are usually defined as

$$
\begin{aligned}
\left\langle\bar{K}^{0}\left|O_{1}(\mu)\right| K^{0}\right\rangle & =\frac{8}{3} M_{K}^{2} f_{K}^{2} B_{1}(\mu) \\
\left\langle\bar{K}^{0}\left|O_{i}(\mu)\right| K^{0}\right\rangle & =\frac{C_{i}}{3}\left(\frac{M_{K}^{2} f_{K}}{m_{s}(\mu)+m_{d}(\mu)}\right)^{2} B_{i}(\mu)
\end{aligned}
$$

where $C_{i}=-5,1,6,2$ for $(i=2, \ldots, 5)$. In (2), $\left\langle\bar{K}^{0}\left|O_{1}\right| K^{0}\right\rangle$ is parameterized in terms of well-known experimental quantities and $B_{1}(\mu)$ $\left(B_{K}(\mu) \equiv B_{1}(\mu)\right)$. On the contrary, $\left\langle\bar{K}^{0}\left|O_{i}\right| K^{0}\right\rangle$ $(i=2, \ldots, 5)$ depend quadratically on the quark masses in (2), while they are expected to remain finite in the chiral limit and depend only linearly on the quark masses. Contrary to $f_{K}, M_{K}$, etc., quark masses can not be directly measured by experiments and the present accuracy in their determination is still rather poor. Therefore, whereas for $O_{1}$ we introduce $B_{K}$ as an alias of the matrix element, by using (2) we replace each of the "SUSY" matrix elements with 2 unknown quantities, i.e. the $B$-parameter and $m_{s}+m_{d}$. To 
Table 1

Matrix elements in $G e V^{4}$ at the renormalization scale $\mu=2$ GeV in the RI scheme obtained with the new parameterization and the conventional one in ref. [3] on the same set of data.

\begin{tabular}{||c|c|c||c|c||}
\hline \hline & \multicolumn{2}{|c||}{ New } & \multicolumn{2}{c||}{ Old } \\
\hline$\left\langle O_{i}\right\rangle$ & $\begin{array}{c}\beta=6.0 \\
\text { this work }\end{array}$ & $\begin{array}{c}\beta=6.2 \\
\text { this work }\end{array}$ & $\begin{array}{c}\beta=6.0 \\
{[9]}\end{array}$ & $\beta=6.2$ \\
& {$[9]$} \\
\hline \hline$\left\langle O_{1}\right\rangle$ & $0.012(2)$ & $0.011(3)$ & $0.012(2)$ & $0.011(3)$ \\
$B_{1}$ & $0.70(15)$ & $0.68(21)$ & $0.70(15)$ & $0.68(21)$ \\
\hline$\left\langle O_{2}\right\rangle$ & $-0.079(10)$ & $-0.074(8)$ & $-0.073(15)$ & $-0.073(15)$ \\
$B_{2}$ & $0.72(9)$ & $0.67(7)$ & $0.66(3)$ & $0.66(4)$ \\
\hline$\left\langle O_{3}\right\rangle$ & $0.027(2)$ & $0.021(3)$ & $0.025(5)$ & $0.022(5)$ \\
$B_{3}$ & $1.21(10)$ & $0.95(15)$ & $1.12(7)$ & $0.98(12)$ \\
\hline$\left\langle O_{4}\right\rangle$ & $0.151(7)$ & $0.133(12)$ & $0.139(28)$ & $0.133(28)$ \\
$B_{4}$ & $1.15(5)$ & $1.00(9)$ & $1.05(3)$ & $1.01(6)$ \\
\hline$\left\langle O_{5}\right\rangle$ & $0.039(3)$ & $0.029(5)$ & $0.035(7)$ & $0.029(7)$ \\
$B_{5}$ & $0.88(6)$ & $0.66(11)$ & $0.79(6)$ & $0.67(10)$ \\
\hline$\left\langle O_{7}^{3 / 2}\right\rangle$ & $0.019(2)$ & $0.011(3)$ & $0.020(5)$ & $0.014(5)$ \\
$B_{7}^{3 / 2}$ & $0.65(5)$ & $0.38(11)$ & $0.68(7)$ & $0.46(13)$ \\
\hline$\left\langle O_{8}^{3 / 2}\right\rangle$ & $0.082(4)$ & $0.068(8)$ & $0.092(19)$ & $0.087(19)$ \\
$B_{8}^{3 / 2}$ & $0.92(5)$ & $0.77(9)$ & $1.04(4)$ & $0.98(8)$ \\
\hline \hline
\end{tabular}

Table 2

RGI Matrix elements in GeV $V^{4}$ computed as in Eq. (10) with $\alpha_{s}^{n_{f}=4}$.

\begin{tabular}{||c|c|c||}
\hline \hline$\left\langle O_{i}^{R G T}\right\rangle$ & $\beta=6.0$ & $\beta=6.2$ \\
\hline \hline$\left\langle O_{1}^{R G T}\right\rangle$ & $0.017(3)$ & $0.016(4)$ \\
\hline$\left\langle O_{2}^{R G T}\right\rangle$ & $-0.051(7)$ & $-0.048(6)$ \\
\hline$\left\langle O_{3}^{R G T}\right\rangle$ & $0.005(7)$ & $-0.004(7)$ \\
\hline$\left\langle O_{4}^{R G T}\right\rangle$ & $0.072(3)$ & $0.063(6)$ \\
\hline$\left\langle O_{5}^{R G T}\right\rangle$ & $0.043(3)$ & $0.032(5)$ \\
\hline \hline
\end{tabular}

overcome these problems, we propose the following new parameterization of $\Delta S=2$ operators

$$
\begin{aligned}
\left\langle\bar{K}^{0}\left|O_{1}(\mu)\right| K^{0}\right\rangle & =\frac{8}{3} M_{K}^{2} f_{K}^{2} B_{1}(\mu), \\
\left\langle\bar{K}^{0}\left|O_{i}(\mu)\right| K^{0}\right\rangle & =M_{K^{*}}^{2} f_{K}^{2} \tilde{B}_{i}(\mu) .
\end{aligned}
$$

The $\tilde{B}_{i}(\mu)$-parameters are still dimensionless quantities and can be computed on the lattice by studying appropriate ratios of three- and twopoint functions [10]. By simply using them, we have eliminated any fictitious reference to the quark masses, hence reducing the systematic errors on the corresponding physical amplitudes. An alternative parameterization, not used here, which can be useful in the future is reported in
10]. The VSA and B-parameters are also used for matrix elements of operators which enter the $\Delta S=1$ effective Hamiltonian. Notice that this "conventional" parameterization is the only responsible for the apparent quadratic dependence of $\epsilon^{\prime} / \epsilon$ on the quark masses. This introduces a redundant source of systematic error which can be avoided by parameterizing the matrix elements in terms of measured experimental quantities and therefore a better determination of the strange quark mass $m_{s}(\mu)$ will not improve our theoretical knowledge of $\epsilon^{\prime} / \epsilon$. In this work we have computed the matrix elements $\left\langle\pi\left|O_{i}^{3 / 2}\right| K\right\rangle$ of the four fermion operators $O_{i}^{3 / 2}(i=7,8,9)$ which contribute to the $\Delta I=3 / 2$ sector of $\epsilon^{\prime} / \epsilon$. In the chiral limit $\left\langle\pi \pi\left|O_{i}^{3 / 2}\right| K\right\rangle$ can be obtained, using soft pion theorems, from $\left\langle\pi^{+}\left|O_{i}^{3 / 2}\right| K^{+}\right\rangle$. For degenerate quark masses, $m_{s}=m_{d}=m$, and in the chiral limit, we find

$$
\begin{aligned}
\lim _{m \rightarrow 0}\left\langle\pi^{+}\left|O_{7}^{3 / 2}\right| K^{+}\right\rangle & =-M_{\rho}^{2} f_{\pi}^{2} \lim _{m \rightarrow 0} \tilde{B}_{5}(\mu) \\
\lim _{m \rightarrow 0}\left\langle\pi^{+}\left|O_{8}^{3 / 2}\right| K^{+}\right\rangle & =-M_{\rho}^{2} f_{\pi}^{2} \lim _{m \rightarrow 0} \tilde{B}_{4}(\mu) \\
\lim _{m \rightarrow 0}\left\langle\pi^{+}\left|O_{9}^{3 / 2}\right| K^{+}\right\rangle & =\frac{8}{3} M_{\pi}^{2} f_{\pi}^{2} \lim _{m \rightarrow 0} B_{1}(\mu) .
\end{aligned}
$$

\section{Renormalization Group Invariant Oper-} ators

Physical amplitudes can be written as

$$
\left\langle F\left|\mathcal{H}_{e f f}\right| I\right\rangle=\langle F|\vec{O}(\mu)| I\rangle \cdot \vec{C}(\mu),
$$

where $\vec{O}(\mu) \equiv\left(O_{1}(\mu), \ldots, O_{N}(\mu)\right)$ is the operator basis (for example the one in (1) for the $\Delta S=2$ ) and $\vec{C}(\mu)$ the corresponding Wilson coefficients represented as a column vector. $\vec{C}(\mu)$ is expressed in terms of its counter-part, computed at a large scale $M$, through the renormalization-group evolution matrix $\hat{W}[\mu, M]$

$\vec{C}(\mu)=\hat{W}[\mu, M] \vec{C}(M)$,

where the initial conditions $\vec{C}(M)$, are obtained by perturbative matching of the full theory to the effective one at the scale $M$ where all the heavy particles have been removed. $\hat{W}[\mu, M]$ can be written as (see for example 11])

$\hat{W}[\mu, M]=\hat{M}[\mu] \hat{U}[\mu, M] \hat{M}^{-1}[M]$, 
where $\hat{U}=\left(\alpha_{s}(M) / \alpha_{s}(\mu)\right)^{\left(\gamma_{O}^{(0) T} / 2 \beta_{0}\right)}$ is the leading-order evolution matrix and $M(\mu)$ is a NLO matrix defined in [11]. The Wilson coefficients $\vec{C}(\mu)$ and the renormalized operators $\vec{O}(\mu)$ are usually defined in a given scheme, at a fixed renormalization scale $\mu$, and they depend on the renormalization scheme and scale in such a way that only $H_{\text {eff }}$ is scheme and scale independent. To simplify the matching procedure, we propose a Renormalization Group Invariant (RGI) definition of Wilson coefficients and composite operators which generalizes what is usually done for $B_{K}$ and for quark masses. We define

$\hat{w}^{-1}[\mu] \equiv \hat{M}[\mu]\left[\alpha_{s}(\mu)\right]^{-\hat{\gamma}_{O}^{(0) T} / 2 \beta_{0}}$,

and using Eqs. (6) and (7) we obtain

$\hat{W}[\mu, M]=\hat{w}^{-1}[\mu] \hat{w}[M]$.

The effective Hamiltonian (位) can be written as $\mathcal{H}_{\text {eff }}=\vec{O}^{R G I} \cdot \vec{C}^{R G I}$, where

$\vec{C}^{R G I}=\hat{w}[M] \vec{C}(M), \vec{O}^{R G I}=\vec{O}(\mu) \cdot \hat{w}^{-1}[\mu]$

$\vec{C}^{R G I}$ and $\vec{O}^{R G I}$ are scheme and scale independent at the order we are working. Therefore the effective Hamiltonian is splitted in terms which are individually scheme and scale independent. This procedure is generalizable to any effective weak Hamiltonian. The RGI $\tilde{B}$-parameters can be defined as

$\tilde{B}_{i}^{R G I}=\sum_{j} \tilde{B}_{j}(\mu) w(\mu)_{j i}^{-1}$.

\section{Numerical results}

All details concerning the extraction of matrix elements from correlation functions and the computation of the non-perturbative renormalization constants of lattice operators can be found in [6, 9, 10]. In this talk we report the results obtained in 10]. The simulations have been performed at $\beta=6.0$ (460 configurations) and 6.2 (200 configurations) with the tree-level Clover action, for several values of the quark masses and for different meson momenta. The main results we have obtained for $\Delta S=2$ and $\Delta I=3 / 2$ matrix elements and their comparison with the results in [9] are reported in Tables 1 and 2. It is interesting to note, as expected from chiral perturbation theory, that matrix elements of $\Delta S=2$
SUSY operators are enhanced respect to the SM one by a factor $2-12$ at $\mu=2 \mathrm{GeV}$. In Figure 1

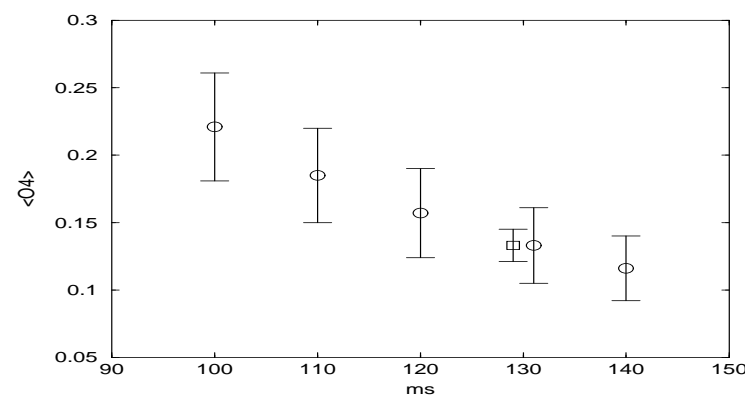

Figure 1. $\left\langle\bar{K}^{0}\left|O_{4}\right| K^{0}\right\rangle\left(\mathrm{GeV}^{4}\right)$ with the new (square) and old (circle) parameterization as a function of the strange quark mass (in $\overline{\mathrm{MS}}$ at $\mu=2 \mathrm{GeV}$ ) used to obtain the full matrix element from the $B$ parameter.

we show the strong dependence of $\left\langle\bar{K}^{0}\left|O_{4}\right| K^{0}\right\rangle$ on the strange quark mass when the "conventional" parameterization (2) is used, to be compared with the result obtained with the new parameterization. The results for the analogous $\Delta C=2$ and $\Delta B=2$ matrix elements presented at the conference are reported in [12]. Although we have data at two different values of the lattice spacing, the statistical errors, and the uncertainties in the extraction of the matrix elements, are too large to enable any extrapolation to the continuum limit. For this reason, the best estimate of the central values of the $B$-parameters can be obtained by averaging the results obtained at the two values of $\beta$ [10]. As far as the errors are concerned we take the largest of the two statistical errors.

\section{REFERENCES}

1. N. Cabibbo et al.,Nucl.Phys. B244(1984)381.

2. R.Brower et al.,Phys.Rev.Lett. 53(1984)1318.

3. C. Bernard in Argonne 1984 p.85.

4. M.Bochicchio et al.Nucl.Phys.B262(1985)331

5. S. Sharpe et al.,Nucl.Phys. B286(1987)253.

6. A. Donini et al.,Eur.Phys.J. C10(1999)121 and ref. therein.

7. L. Lellouch,hep-ph/9906497 and ref. therein.

8. T.Bhattacharya et al.Ph.Rev.D55(1997)4036

9. C. R. Allton et al., Phys. Lett. B453(1999)30.

10. A. Donini et al., in preparation.

11. M. Ciuchini et al.,Nucl.Phys. B523(1998)501.

12. L. Conti et al., in preparation. 